PROCEEDINGS OF THE

AMERICAN MATHEMATICAL SOCIETY

Volume 132, Number 10, Pages 2857-2858

S 0002-9939(04)07334-4

Article electronically published on June 2, 2004

\title{
A REMARK ON A THEOREM BY DELIGNE
}

\author{
M. VAN DEN BERGH
}

(Communicated by Lance W. Small)

\begin{abstract}
We give a proof avoiding spectral sequences of Deligne's decomposition theorem for objects in a triangulated category admitting a Lefschetz homomorphism.
\end{abstract}

Below $\mathcal{A}$ is a triangulated category equipped with a bounded t-structure. In addition, $\mathcal{A}$ will be equipped with an auto-equivalence $A \mapsto A(1)$ compatible with the t-structure.

In [1, 2] Deligne proves the following result:

Theorem 1. Let $A$ be an object of $\mathcal{A}$ equipped with an endomorphism $\phi: A[-1] \rightarrow$ $A[1](1)$ such that its iterates $\phi^{n}: A[-n] \rightarrow A[n](n)$ induce isomorphisms $H^{-n}(A) \rightarrow$ $H^{n}(A)(n)$. Then there exists an isomorphism $A \cong \bigoplus_{k} H^{-k}(A)[k]$.

Deligne's proof is slightly indirect. He first shows that a decomposition as asserted in Theorem 1 exists if and only if for every cohomological functor $T: \mathcal{A} \rightarrow \mathcal{C}$ to an abelian category the resulting spectral sequence

$$
E_{2}^{p q}: T^{p}\left(H^{q}(A)\right) \Rightarrow T^{p+q}(A)
$$

degenerates. He then proceeds to show that (1) does indeed degenerate.

The aim of this note is to give a proof of Theorem 1 that avoids the use of spectral sequences.

Let $A, \phi$ be as in the statement of Theorem 1 . We start with the following statement:

$\left(\operatorname{Hyp}_{n}\right) \quad A \cong A_{n} \oplus\left(\bigoplus_{|k|>n} H^{-k}(A)[k]\right)$ with $A_{n} \in \mathcal{A}^{[-n, n]}$.

By the boundedness of the t-structure, $\left(\operatorname{Hyp}_{n}\right)$ is true for $n \gg 0$. We need to show that it is true for $n=0$, for which we use descending induction on $n$.

Assume $\left(\operatorname{Hyp}_{n}\right)$ is true for $n \geq 1$. We will show that $\left(\operatorname{Hyp}_{n-1}\right)$ is also true. Without loss of generality, we may assume that the isomorphism in the statement of $\left(\operatorname{Hyp}_{n}\right)$ is an equality. Let $i: A_{n} \rightarrow A, p: A \rightarrow A_{n}$ be, respectively, the inclusion and the projection map. They induce identifications $H^{q}(A)=H^{q}\left(A_{n}\right)$ for $|q| \leq n$. Let $\alpha$ be the composition of the following maps:

$$
H^{-n}(A) \rightarrow A_{n}[-n] \stackrel{i}{\rightarrow} A[-n] \stackrel{\phi^{n}}{\longrightarrow} A[n](n) \stackrel{p}{\rightarrow} A_{n}[n](n) \rightarrow H^{n}(A)(n)
$$

Received by the editors February 11, 2003.

2000 Mathematics Subject Classification. Primary 18E30.

Key words and phrases. Triangulated categories, spectral sequence.

The author is a senior researcher at the FWO. 
where the first and last map are obtained from the canonical maps $H^{-n}\left(A_{n}\right)[n] \rightarrow$ $A_{n} \rightarrow H^{n}\left(A_{n}\right)[-n]$ that exist because $A_{n} \in \mathcal{A}^{[-n, n]}$.

Applying $H^{0}(-)$ and the hypotheses we find that $\alpha=H^{0}\left(\phi^{n}\right)$, and hence it is an isomorphism. Composing arrows we find that $\alpha$ is also the composition of maps

$$
\begin{aligned}
& H^{-n}(A) \rightarrow A_{n}[-n] \rightarrow H^{n}(A)(n), \\
& H^{-n}(A) \rightarrow A_{n}[n](n) \rightarrow H^{n}(A)(n)
\end{aligned}
$$

inducing isomorphisms on $H^{0}$.

From (21) it follows that

$$
A_{n}[-n] \cong H^{-n}(A) \oplus C
$$

for some $C \in \mathcal{A}^{[1,2 n]}$. Shifting and substituting in (3) we deduce that $\alpha$ is a composition

$$
H^{-n}(A) \rightarrow H^{-n}(A)[2 n](n) \oplus C[2 n](n) \rightarrow H^{n}(A)(n) .
$$

Since $\operatorname{Hom}_{\mathcal{A}}\left(H^{-n}(A)[2 n](n), H^{n}(A)(n)\right)=0$, we see that $\alpha$ is actually a composition

$$
H^{-n}(A) \rightarrow C[2 n](n) \rightarrow H^{n}(A)(n),
$$

and these maps still induce isomorphisms in degree zero. Thus

$$
C[2 n](n) \cong H^{n}(A)(n) \oplus D
$$

for $D \in \mathcal{A}^{[-2 n+1,-1]}$. Shifting and substituting in (4) yields a decomposition

$$
A_{n} \cong H^{-n}(A)[n] \oplus H^{n}(A)[-n] \oplus D[-n](-n) .
$$

Putting $A_{n-1}=D[-n](-n)$ finishes the induction step and the proof.

Remark 2. It follows from the above proof that the decomposition asserted in Theorem[still exists if we have maps $\phi_{n}: A[-n] \rightarrow A[n](n)$ inducing isomorphisms $H^{-n}(A) \rightarrow H^{n}(A)(n)$ that are not necessarily powers of a fixed $\phi: A[-1] \rightarrow$ $A[1](1)$. However, I have no example where this extra generality applies.

Remark 3. In [2] Deligne constructs several canonical isomorphisms

$$
A \cong \bigoplus_{k} H^{-k}(A)[k] .
$$

We have not tried to duplicate these constructions with our approach.

\section{REFERENCES}

[1] P. Deligne, Théorème de Lefschetz et critères de dégénérescence de suites spectrales, Inst. Hautes Études Sci. Publ. Math., no. 35 (1968), 259-278. MR 39:5582

[2] P. Deligne, Décompositions dans la catégorie dérivée, Motives (Seattle, WA, 1991), Proc. Sympos. Pure Math., vol. 55, Part 1, Amer. Math. Soc., Providence, RI, 1994, pp. 115-128. MR 95h:18013

Departement WNi, Limburgs Universitair Centrum, 3590 Diepenbeek, Belgium

E-mail address: vdbergh@luc.ac.be 